\title{
ANÁLISE DOS FATORES DE RISCO PARA DOENÇAS CRÔNICAS NÃO TRANSMISSIVVIS: estudo com colaboradores de uma instituição privada
}

\author{
ANALYSIS OF THE RISK FACTORS FOR CHRONIC \\ NON-COMMUNICABLE DISEASE: \\ study with employees of a private institution
}

\section{Veronilde da Silva Oliveira', Paula Christianne Gomes Gouveia Souto Maia², Joselito Santos ${ }^{3}$, Milena Nunes Alves de Sousa ${ }^{4}$}

\section{RESUMO}

Objetivo: Analisar os fatores de risco para doenças crônicas não transmissiveis entre colaboradores de uma instituição privada. Trata-se de um estudo descritivo, documental, com abordagem quantitativa, realizado a partir de uma investigação em um banco de dados. Os dados foram tabulados por meio do Software de Gestão - Infomed e apresentados em gráficos e tabelas. O tratamento dos dados se deu através de análise estatística descritiva. Os resultados evidenciaram alta prevalência de excesso de peso (61\%), tabagismo (2\%), etilismo (58\%), sedentarismo (72\%) e alimentação não saudável (83\%). Nas doenças já instaladas evidenciou-se hipertensão arterial sistêmica em 10\% dos entrevistados, $11 \%$ com dislipidemia, $7 \%$ têm problemas respiratórios, $2,8 \%$ são diabéticos e $0,8 \%$ têm depressão. Foi possível constatar que os colaboradores apresentam alto risco para o desenvolvimento de doenças crônicas não transmissíveis em decorrência da alta prevalência dos fatores de risco observados no estudo.

Descritores: Saúde do Trabalhador; Saúde Suplementar; Fatores de Risco.

\section{ABSTRACT}

Objective: To analyze the risk factors for chronic diseases among employees of a private institution. This is a descriptive study, documentary, with a quantitative approach, carried out from an investigation into a database. The data were tabulated through Management Software - Infomed and presented in graphs and tables. The processing of data through descriptive statistical analysis. The results showed a high prevalence of overweight $(61 \%)$, smoking (2\%), alcohol $(58 \%)$, physical inactivity $(72 \%)$ and unhealthy diet (83\%). The already installed disease showed up arterial hypertension in $10 \%$ of subjects, $11 \%$ had dyslipidemia, $7 \%$ have respiratory problems, diabetics are $2.8 \%$ and $0.8 \%$ have depression. It was found that the employees have high risk for developing chronic non-communicable diseases due to the high prevalence of risk factors observed in the study.

Descriptors: Occupational Health; Supplemental Health; Risk Factors.
${ }^{1}$ Mestre em Saúde Coletiva e Gestão Hospitalar pela Fundação Universitária de Apoio ao Ensino Pesquisa e Extensão (FURNE), Campina Grande, PB, Brasil.

${ }^{2}$ Doutoranda em Ciências da Saúde pela Faculdade de Medicina do ABC (FMABC), Santo André, SP, Brasil.

${ }^{3}$ Doutor em Ciências Sociais pela Universidade Federal do Rio Grande do Norte (UFRN), Natal, RN, Brasil.

${ }^{4}$ Pós-Doutora em Promoção de Saúde pela Universidade de Franca (UNIFRAN), Franca, SP, Brasil. 


\section{Introdução}

As doenças crônicas não transmissíveis (DCNT) são as principais causas de óbitos no mundo e têm gerado elevado número de mortes prematuras, perda de qualidade de vida $(\mathrm{QV})$ com alto grau de limitação nas atividades de trabalho e de lazer, além de impactos econômicos para as famílias, comunidades e a sociedade em geral, agravando as iniquidades e aumentando a pobreza ${ }^{1-2}$.

Apesar do rápido crescimento das DCNT, seu impacto pode ser revertido por meio de intervenções amplas e custo-efetivas de promoção de saúde, para redução de seus fatores de risco e pela melhoria da atenção à saúde, detecção precoce e tratamento oportuno ${ }^{3}$.

Das 57 milhões de mortes no mundo em 2008, 36 milhões - ou 63,0\% - aconteceram em razão das DCNT, com destaque para as doenças do aparelho circulatório, diabetes mellitus (DM), câncer e doença respiratória crônica. Cerca de $80,0 \%$ das mortes por DCNT ocorrem em países de baixa ou média renda, onde $29,0 \%$ das pessoas têm menos de 60 anos de idade. Nos países de renda alta, apenas $13,0 \%$ são mortes precoces 2 .

No Brasil, como em outros países, as DCNT constituem o problema de saúde de maior magnitude ${ }^{1,4}$. São responsáveis por $72,0 \%$ das causas de óbitos, com destaque para doenças do aparelho circulatório (DAC) (31,3\%), câncer (16,3\%), DM (5,2\%) e doença respiratória crônica $(5,8 \%)^{4}$ e atingem indivíduos de todas as camadas socioeconômicas e, de forma mais intensa, aqueles pertencentes a grupos vulneráveis, como os idosos, os de baixa escolaridade e renda ${ }^{3}$.

Existem evidências de que a exposição crônica a condições estressantes de trabalho, como aumento da demanda de atividade laboral; ambientes de trabalho com frágil nível organizacional, com altos níveis de ruído e elevadas temperaturas; longas jornadas de trabalho; e trabalho por turno podem ser responsáveis pelos aumentos expressivos e persistentes no aparecimento das DCNT. Fatores ambientais, como estilo de vida e dieta, desempenham papel fundamental, ou seja, a genética não é uma rota determinante e cada vez mais pode ser "manipulada" em beneficio da saúde 5 .

A exposição aos fatores de risco e de proteção para DCNT são diferentes entre homens e mulheres. Segundo dados do "Sistema de Monitoramento de Fatores de Risco e Proteção para DCNT por meio de Inquérito Telefônico 2010", os cinco fatores de risco selecionados para estudo (tabagismo, consumo abusivo de bebidas alcoólicas, excesso de peso, consumo de carnes com excesso de gordura e inatividade física) foram mais frequentes em homens do que em mulheres. Já o fator de proteção consumo regular de frutas e hortaliças foi mais comum em mulheres do que em homens ${ }^{6}$.

As elevadas taxas de morbimortalidade por DCNT acarretam em altos custos econômicos e sociais em decorrência dos gastos com cuidados médicos e uso dos serviços de saúde, demanda aumentada por pensões por incapacidade e compensação do seguro social. Isto ocorre devido ao grande número de pessoas que se aposentam precocemente ou que se tornam incapazes para o trabalho de forma definitiva ou temporária, às elevadas taxas de absenteísmo, e à diminuição da produtividade no trabalho. Tais ônus afetam não só os indivíduos acometidos pelas doenças, como também suas famílias, o governo, a sociedade e as empresas ${ }^{7}$.

Os principais fatores de risco para as DCNT são o tabaco, a alimentação não saudável, a inatividade física e o consumo nocivo de álcool, responsáveis, em grande parte, pela epidemia de sobrepeso e obesidade, pela elevada prevalência de hipertensão arterial sistêmica (HAS) e pelo colesterol alto ${ }^{3}$.

As doenças cardiovasculares (DCV) são, atualmente, as causas mais comuns de morbimortalidade no mundo. $\mathrm{Na}$ perspectiva da prevenção de doenças e agravos, tornam-se fundamentais ações que criem ambientes favoráveis à saúde e favoreçam escolhas saudáveis ${ }^{8}$. Anualmente, a cardiopatia isquêmica, acidentes vasculares cerebrais (AVC), HAS e outras cardiopatias são responsáveis por 15,9 milhões de óbitos 9 .

O Brasil, por meio de diferentes inquéritos de saúde, vem construindo bases de dados para o monitoramento contínuo dos fatores de risco e de proteção das DCNT. As campanhas de detecção de suspeitos de HAS e DM realizadas nos anos de 2001 e 2002 no Brasil, parte do Plano de Reorganização da Atenção à HAS e ao DM do Ministério da Saúde (MS), configuraram a primeira iniciativa mundial de realização de uma campanha populacional massiva para 0 rastreamento de $\mathrm{DCNT}^{10}$.

Na saúde privada os estudos sobre os fatores de risco entre usuários de planos de saúde são pouco frequentes. Pesquisa contemplou a associação entre filiação aos planos de saúde e estilos de vida e uso de serviços preventivos em Belo Horizonte e encontrou que os indivíduos que possuíam planos de saúde apresentaram hábitos mais saudáveis de vida, sendo também a prevalência de uso de serviços preventivos mais altos ${ }^{11}$. 
Diante do contexto, indagam-se quais os fatores de risco para DCNT entre colaboradores de uma instituição privada? A identificação prévia destes elementos nos beneficiários pode auxiliar na promoção da modificação de comportamento, corroborando com a redução de riscos de doença e custos relacionados. As ações permitem uma gestão mais customizada e eficiente dos benefícios oferecidos e, assim, atinge o objetivo de melhorar a saúde e a QV dos colaboradores/beneficiários.

Assim sendo, objetiva-se analisar os fatores de risco para DCNT entre colaboradores de uma instituição privada.

\section{Metodologia}

Estudo descritivo, documental, com abordagem quantitativa. O local de escolha para o desenvolvimento deste estudo foi o banco de dados da Unimed Norte Nordeste - Federação Interfederativa de Trabalhos Médicos. A mesma adotou a estratégia de promoção da saúde e prevenção de doença, em fevereiro de 2013, criando o Núcleo de Atenção à Saúde, criado a partir da necessidade de concepção de um novo modelo de atenção à saúde, para assistir e orientar de maneira integral os beneficiários e colaboradores-beneficiários ${ }^{12}$.

A população constituiu-se de 278 beneficiários-colaboradores que atuam desde a operacionalização até a gestão. Neste estudo foi utilizada a amostragem intencional para selecionar os sujeitos da amostra. Essa forma de seleção pode ser traduzida como aquela em que o pesquisador seleciona os sujeitos que considera relevantes para seu estudo ${ }^{13}$. Portanto, participaram do estudo 256 indivíduos (92,08\%).

Os critérios de inclusão atenderam aos seguintes parâmetros: ser colaborador ativo independente do cargo e participar dos programas de promoção à saúde e prevenção de doenças disponibilizadas pela empresa. Excluíram os cadastros incompletos.

A coleta de dados ocorreu entre julho e setembro de 2015 e os dados dispostos no banco de dados da Unimed Norte Nordeste - Federação Interfederativa de Trabalhos Médicos foi coletada em 2015, a partir de um questionário aplicado durante entrevistas, o qual abordava as características demográficas e socioeconômicas, padrão de alimentação e de atividade física, peso e altura, estes possibilitando o cálculo do Índice de Massa Corporal (IMC), hábito tabagista e de consumo de bebida alcoólica, autoavaliação do estado de saúde, prevenção de câncer e morbidade referida ${ }^{14}$. As variáveis do estudo foram:

- Tabagismo: proporção de indivíduos que fumam, independente da frequência e intensidade do hábito de fumar. Ex-fumantes: proporção de indivíduos que já fumaram e não fumam mais.

- Consumo de bebidas alcoólicas: frequência de uso da bebida alcoólica.

- Excesso de peso: proporção de indivíduos com IMC igual ou superior a $25 \mathrm{Kg} / \mathrm{m}^{2}$. Para calcular o IMC, divide-se o peso pela altura ao quadrado. Obesidade: proporção de indivíduos com IMC igual ou superior a $30 \mathrm{Kg} / \mathrm{m}^{2}$. Para calcular o IMC, divide-se o peso pela altura ao quadrado.

- Alimentação Saudável: proporção de indivíduos com alimentação saudável. Considera-se alimentação saudável o indivíduo que come frutas de duas a três porções diárias, legumes e verduras no mínimo três porções diárias. Aquele indivíduo que ao consumir carne retira a gordura ou pele; ou aquele que consume alimentos desnatados e não comem frituras durante os cinco a sete dias na semana.

- Prática suficiente de atividade física: proporção de indivíduos que praticam atividades físicas acima de 30 minutos diários em três ou mais dias por semana.

- Morbidade autorreferida: proporção de indivíduos que relatam diagnóstico médico de: HAS, DM, dislipidemia, doenças cardíacas, depressão, câncer, doenças respiratórias.

- Autoavaliação do estado de saúde como Fraca: proporção de indivíduos que consideram seu estado de saúde como fraca. Autoavaliação do estado de saúde como Regular: proporção de indivíduos que consideram seu estado de saúde como regular. Autoavaliação do estado de saúde como Péssima: proporção de indivíduos que consideram seu estado de saúde como péssima. Autoavaliação do estado de saúde como Boa: proporção de indivíduos que consideram seu estado de saúde como boa. Autoavaliação do estado de saúde como Muito Boa: proporção de indivíduos que consideram seu estado de saúde como muito boa. Autoavaliação do estado de saúde como ótima: proporção de indivíduos que consideram seu estado de saúde como ótima.

- Exame de Papanicolau/Preventivo de Câncer de Colo Uterino (PCCU): proporção de mulheres com idade entre 25 e 59 anos que declaram ter realizado o PCCU alguma vez na vida ou nos três últimos anos. 
- Exame de mamografia: proporção de mulheres com idade entre 50 e 59 anos que declararam ter realizado exame de mamografia alguma vez na vida e nos últimos dois anos.

Para análise dos dados, adotou-se a estatística descritiva por meio de medidas de tendência central (MTC) (Média Aritmética [MA], Moda [Mo] e Mediana [Me]) e medidas de dispersão (MD) (Desvio Padrão [DP]), sendo-os expressos em frequência absoluta (n) e relativa/percentual (\%).

O estudo obedeceu aos preceitos éticos da Resolução n 466/2012, do Conselho Nacional de Saúde (CNS), na qual regulamenta a pesquisa envolvendo seres humanos. 0 projeto de pesquisa foi apreciado e aprovado pelo Comitê de Ética em Pesquisa da Fundação de Apoio ao Ensino, à Pesquisa e à Extensão (CEP FURNE), Protocolo n 1.153.211/2015, Certificado de Apresentação para Apreciação Ética (CAAE) nº 46842715.1.0000.5693. O Termo de Consentimento Livre e Esclarecido (TCLE) foi assinado por todos os participantes.

\section{Resultados}

A tabela 1 apresenta a distribuição segundo a idade dos colaboradores. Verificou-se que a média de idade dos profissionais é de 34 a 38 anos, com idade mínima de 18 anos e a idade máxima 70 anos, sendo $54 \%$ do sexo feminino e $46 \%$ do sexo masculino.

Tabela 1 - Perfil etário dos colaboradores beneficiários de planos de saúde conforme diferenciação do gênero

\begin{tabular}{c|c|c|c|c|c|c|c|c|c|c}
\hline \multirow{2}{*}{ Gênero } & \multicolumn{10}{c}{ Faixa Etária } \\
\cline { 2 - 13 } & $\mathbf{0 - 1 8}$ & $\mathbf{1 9 - 2 3}$ & $\mathbf{2 4 - 2 8}$ & $\mathbf{2 9 - 3 3}$ & $\mathbf{3 4 - 3 8}$ & $\mathbf{3 9 - 4 4}$ & $\mathbf{4 5 - 5 3}$ & $\mathbf{5 4 - 5 8}$ & $\mathbf{5 9 - 1 2 0}$ & Total \\
\hline Feminino & - & 11 & 23 & 28 & 35 & 23 & 15 & 02 & 02 & 139 \\
\hline Masculino & 01 & 08 & 22 & 22 & 24 & 12 & 16 & 07 & 05 & 117 \\
\hline
\end{tabular}

Fonte: Dados da pesquisa, 2015.

Na tabela 2 têm-se os dados sobre a realização de PCCU e mamografia por ano, em que se constatou haver uma queda no ano de 2014, e após um aumento considerável na realização dos exames preventivos no ano de 2015. No exame de mamografia, a faixa etária que mais realizou está entre 39 e 44 anos; no PCCU, as maiores realizações de exames foram entre as idades de 34 a 38 anos. Observa-se também nestes dados que as mulheres entre 54 a 58 anos se preocupam pouco com a realização desses exames, pois apenas 3\% das participantes realizaram a mamografia e o PCCU nesta faixa etária.

Tabela 2 - Perfil clínico das mulheres que realizaram o PCCU e a mamografia conforme distribuição etária e ano de avaliação

\begin{tabular}{c|c|c|c|c|c|c|c|c}
\hline \multicolumn{7}{c}{ Quantidade por idade X Ano de avaliação } \\
\hline \multirow{2}{*}{ Variável } & $\begin{array}{c}\text { Quantidade por } \\
\text { idade }\end{array}$ & \multicolumn{2}{c}{$\mathbf{2 0 1 3}$} & \multicolumn{2}{c}{$\mathbf{2 0 1 4}$} & \multicolumn{2}{c}{2015} \\
\cline { 2 - 9 } & $\mathbf{n}$ & $\%$ & $\mathbf{n}$ & $\%$ & $\mathbf{n}$ & \% \\
\hline Mamografia Faixa etária (anos) & 11 & - & - & - & - & - & - \\
\hline $19-13$ & 23 & - & - & - & - & - & - \\
\hline $24-28$ & 28 & - & - & - & - & - & - \\
\hline $29-33$ & 35 & 08 & - & 01 & - & 16 & - \\
\hline $34-38$ & 23 & 13 & - & 01 & - & 11 & - \\
\hline $39-44$ & 15 & 13 & - & 03 & - & 15 & - \\
\hline $45-53$ & 02 & 01 & - & - & - & 01 & - \\
\hline $54-58$ & 02 & 01 & - & - & - & 03 & - \\
\hline $59-120$ & $\mathbf{1 3 9}$ & $\mathbf{3 3}$ & $\mathbf{2 4}$ & $\mathbf{0 5}$ & $\mathbf{0 4}$ & $\mathbf{4 6}$ & $\mathbf{3 3}$ \\
\hline Total & & & & & & &
\end{tabular}




\begin{tabular}{|c|c|c|c|c|c|c|c|}
\hline \multicolumn{8}{|c|}{ Quantidade por idade X Ano de avaliação } \\
\hline \multirow{2}{*}{ Variável } & \multirow{2}{*}{$\begin{array}{l}\text { Quantidade por } \\
\text { idade }\end{array}$} & \multicolumn{2}{|c|}{2013} & \multicolumn{2}{|c|}{2014} & \multicolumn{2}{|c|}{2015} \\
\hline & & $\mathbf{n}$ & $\%$ & $\mathbf{n}$ & $\%$ & $\mathbf{n}$ & $\%$ \\
\hline \multicolumn{8}{|c|}{ PCCU Faixa etária (anos) } \\
\hline 19-13 & 11 & - & - & - & - & - & - \\
\hline $24-28$ & 23 & 04 & - & 01 & - & 17 & - \\
\hline $29-33$ & 28 & 11 & - & 04 & - & 28 & - \\
\hline $34-38$ & 35 & 16 & - & 05 & - & 33 & - \\
\hline $39-44$ & 23 & 15 & - & 01 & - & 23 & - \\
\hline $45-53$ & 15 & 10 & - & 03 & - & 15 & - \\
\hline 54-58 & 02 & 01 & - & - & - & 01 & - \\
\hline 59-120 & 02 & - & - & - & - & 01 & - \\
\hline Total & 139 & 57 & 41 & 14 & 10 & 118 & 85 \\
\hline
\end{tabular}

Fonte: Dados da pesquisa, 2015.

Na tabela 3, verifica-se que $55 \%$ dos pesquisados responderam ter boa saúde boa.

Tabela 3 - Análise dos colaboradores quanto à avaliação do perfil de percepção sobre sua saúde

\begin{tabular}{|c|c|c|c|c|c|c|c|c|c|c|c|}
\hline \multicolumn{2}{|c|}{ Variáveis } & \multicolumn{2}{|c|}{ Excelente } & \multicolumn{2}{|c|}{ Muito Boa } & \multicolumn{2}{|c|}{ Boa } & \multicolumn{2}{|c|}{ Fraca } & \multicolumn{2}{|c|}{ Regular } \\
\hline Faixa etária & Sexo & $\mathbf{n}$ & $\%$ & $\mathbf{n}$ & $\%$ & $\mathbf{n}$ & $\%$ & $\mathbf{n}$ & $\%$ & $\mathbf{n}$ & $\%$ \\
\hline \multirow{2}{*}{$0-18$} & $M$ & - & & - & & - & & - & & 01 & \\
\hline & $\mathrm{F}$ & - & & - & & - & & - & & - & \\
\hline \multirow{2}{*}{$19-23$} & M & - & & 01 & & 06 & & - & & 01 & \\
\hline & $\mathrm{F}$ & - & & - & & 06 & & - & & 02 & \\
\hline \multirow{2}{*}{$24-28$} & M & 01 & & 05 & & 12 & & - & & 04 & \\
\hline & $\mathrm{F}$ & 02 & & 05 & & 14 & & 01 & & 05 & \\
\hline \multirow{2}{*}{$29-33$} & M & - & & 06 & & 10 & & 01 & & 04 & \\
\hline & $\mathrm{F}$ & 02 & & 08 & & 16 & & - & & 03 & \\
\hline \multirow{2}{*}{$34-38$} & M & 01 & & 05 & & 12 & & 01 & & 02 & \\
\hline & $\mathrm{F}$ & 01 & & 09 & & 22 & & 01 & & 02 & \\
\hline \multirow{2}{*}{$39-44$} & M & 01 & & 02 & & 04 & & 01 & & 01 & \\
\hline & $\mathrm{F}$ & - & & 05 & & 10 & & 01 & & 06 & \\
\hline \multirow{2}{*}{$45-53$} & M & - & & 01 & & 10 & & 01 & & 02 & \\
\hline & $\mathrm{F}$ & - & & 05 & & 06 & & - & & 02 & \\
\hline \multirow{2}{*}{$54-58$} & M & - & & 01 & & 05 & & - & & - & \\
\hline & $\mathrm{F}$ & - & & - & & 01 & & - & & 01 & \\
\hline \multirow{2}{*}{$59-120$} & M & 02 & & - & & 01 & & - & & 01 & \\
\hline & $\mathrm{F}$ & 01 & & - & & 01 & & - & & - & \\
\hline \multirow{2}{*}{ Total } & $\mathbf{M}$ & 05 & 05 & 21 & 20 & 60 & 57 & 04 & 04 & 15 & 14 \\
\hline & $\mathbf{F}$ & 06 & 04 & 32 & 23 & 76 & 55 & 03 & 02 & 21 & 16 \\
\hline
\end{tabular}

Fonte: Dados da pesquisa, 2015.

Na tabela 4, 2\% afirmaram que são tabagistas, sendo mais homens que mulheres. Já na realização de atividades físicas, o inverso acontece, onde teve um percentual de $44 \%$ de mulheres sedentárias. Quanto ao etilismo, 58\% dos 
avaliados responderam que bebem, sendo $29 \%$ homens e 30\% mulheres. Em relação à alimentação inadequada, $83 \%$ da população têm uma alimentação não saudável.

Tabela 4 - Distribuição da prevalência dos fatores de risco para DCNT entre a população conforme o sexo

\begin{tabular}{|c|c|c|c|c|c|c|c|}
\hline \multirow{2}{*}{\multicolumn{2}{|c|}{ Fatores de risco }} & \multicolumn{4}{|c|}{ Sexo } & \multirow{2}{*}{\multicolumn{2}{|c|}{ Total }} \\
\hline & & \multicolumn{2}{|c|}{ Masculino } & \multicolumn{2}{|c|}{ Feminino } & & \\
\hline & & $\mathbf{n}$ & $\%$ & $\mathbf{n}$ & $\%$ & $\mathbf{n}$ & $\%$ \\
\hline \multirow{2}{*}{ Tabagismo } & Sim & 04 & 02 & 02 & 01 & 06 & 02 \\
\hline & Não & 104 & 42 & 140 & 56 & 244 & 98 \\
\hline \multirow{2}{*}{ Sedentarismo } & Sim & 70 & 28 & 110 & 44 & 180 & 72 \\
\hline & Não & 38 & 15 & 32 & 13 & 70 & 28 \\
\hline \multirow{2}{*}{ Etilismo } & Sim & 72 & 29 & 74 & 30 & 146 & 58 \\
\hline & Não & 36 & 14 & 68 & 27 & 104 & 42 \\
\hline \multirow{2}{*}{$\begin{array}{l}\text { Alimentação } \\
\text { inadequada }\end{array}$} & Sim & 89 & 36 & 118 & 47 & 207 & 83 \\
\hline & Não & 16 & 06 & 27 & 11 & 43 & 17 \\
\hline
\end{tabular}

Fonte: Dados da pesquisa, 2015.

Na tabela $5,1 \%$ dos entrevistados na faixa etária de 29 a 44 anos é fumante, enquanto na faixa etária de 45 a 120 anos apresentou $2 \%$. Com relação ao sedentarismo, etilismo e alimentação inadequada, a faixa etária com maior prevalência de inatividade física está entre os 29 a 44 anos.

Tabela 5 - Distribuição da prevalência dos fatores de risco para DCNT entre a população conforme a faixa etária

\begin{tabular}{|c|c|c|c|c|c|c|c|c|c|}
\hline \multirow{3}{*}{ Fatores de risco } & & \multicolumn{6}{|c|}{ Faixa Etária } & \multirow{2}{*}{\multicolumn{2}{|c|}{ Total }} \\
\hline & & \multicolumn{2}{|c|}{$18-28$} & \multicolumn{2}{|c|}{$29-44$} & \multicolumn{2}{|c|}{$45-120$} & & \\
\hline & & $\mathbf{n}$ & $\%$ & $\mathbf{n}$ & $\%$ & $\mathbf{n}$ & $\%$ & $\mathbf{n}$ & $\%$ \\
\hline \multirow{2}{*}{ Tabagismo } & Sim & 00 & 00 & 02 & 01 & 04 & 02 & 06 & 02 \\
\hline & Não & 65 & 26 & 139 & 56 & 40 & 16 & 244 & 98 \\
\hline \multirow{2}{*}{ Sedentarismo } & Sim & 39 & 16 & 103 & 41 & 38 & 15 & 180 & 72 \\
\hline & Não & 26 & 10 & 38 & 15 & 06 & 02 & 70 & 28 \\
\hline \multirow{2}{*}{ Etilismo } & Sim & 36 & 14 & 81 & 32 & 29 & 12 & 146 & 58 \\
\hline & Não & 29 & 12 & 60 & 24 & 15 & 06 & 104 & 42 \\
\hline \multirow{2}{*}{ Alimentação inadequada } & Sim & 58 & 23 & 113 & 45 & 36 & 14 & 207 & 83 \\
\hline & Não & 05 & 02 & 28 & 11 & 10 & 04 & 43 & 17 \\
\hline
\end{tabular}

Fonte: Dados da pesquisa, 2015.

Na tabela 6 apresenta-se o resultado do IMC, demonstrando que $62 \%$ da população estavam com excesso de peso e os homens apresentaram maior excesso de peso (71\%) que as mulheres (55\%). Entre os indivíduos com mais de 24 anos, o homens também apresentaram maior excesso de peso do que as mulheres. Observou-se que as mulheres na faixa etária entre 24 a 28 anos de idade apresentaram percentual maior de excesso de peso.

O inverso ocorreu entre os homens, em que aqueles entre 29 e 33 anos são os que apresentam o maior percentual de excesso de peso. A obesidade foi de 30\% entre homens na faixa etária de 19 a 44 anos. Nas mulheres, este percentual foi de 18\% na faixa etária de 19 a 38 anos. Independente da faixa etária, 18\% das mulheres estavam obesas e 30\% dos homens. 
Tabela 6 - Perfil de indivíduos com sobrepeso $\left(\mathrm{IMC}>25 \mathrm{~kg} / \mathrm{m}^{2}\right)$ e obesidade (IMC > $\left.30 \mathrm{~kg} / \mathrm{m}^{2}\right)$ conforme a faixa etária.

\begin{tabular}{|c|c|c|c|c|c|c|c|c|c|c|c|}
\hline \multicolumn{12}{|c|}{ FAIXA ETÁRIA } \\
\hline HOMENS & $0-18$ & $19-23$ & $24-28$ & $29-33$ & $34-38$ & $39-44$ & $45-53$ & $54-58$ & $59-120$ & Total & $\%$ \\
\hline Baixo peso & - & - & - & - & - & - & - & - & - & - & - \\
\hline Normal & 01 & 03 & 04 & 08 & 07 & 03 & 05 & 02 & 01 & 34 & 29 \\
\hline Sobrepeso & - & 03 & 09 & 07 & 08 & 06 & 07 & 04 & 04 & 48 & 41 \\
\hline $\begin{array}{c}\text { Obesidade } \\
\text { grau I }\end{array}$ & - & - & 06 & 04 & 04 & - & 04 & 01 & - & 19 & 16 \\
\hline $\begin{array}{c}\text { Obesidade } \\
\text { grau II }\end{array}$ & - & 02 & 03 & 02 & 03 & 02 & - & - & - & 12 & 11 \\
\hline $\begin{array}{c}\text { Obesidade } \\
\text { grau III }\end{array}$ & - & - & - & 01 & 02 & 01 & - & - & - & 04 & 03 \\
\hline \multicolumn{12}{|l|}{ MULHERES } \\
\hline Baixo peso & - & - & 01 & 01 & - & - & - & - & - & 02 & 01 \\
\hline Normal & - & 07 & 10 & 09 & 17 & 07 & 08 & 01 & 02 & 61 & 44 \\
\hline Sobrepeso & - & 03 & 08 & 12 & 13 & 12 & 04 & - & - & 52 & 37 \\
\hline $\begin{array}{c}\text { Obesidade } \\
\text { grau I }\end{array}$ & - & - & 03 & 05 & 04 & 04 & 03 & 01 & - & 20 & 14 \\
\hline $\begin{array}{c}\text { Obesidade } \\
\text { grau II }\end{array}$ & - & - & - & 01 & - & - & - & - & - & 01 & 01 \\
\hline $\begin{array}{c}\text { Obesidade } \\
\text { grau III }\end{array}$ & - & 01 & 01 & - & 01 & - & - & - & - & 03 & 03 \\
\hline
\end{tabular}

Fonte: Dados da pesquisa, 2015.

Quanto à morbidade autorreferida, a proporção de indivíduos que relatam diagnóstico médico para DCNT foi: $11 \%$ relataram dislipidemia, 10\% HAS, 7\% doenças respiratórias, 2,8\% DM e 0,8\% para depressão. No mais, 12,9\% afirmou possuir outras doenças e $55,5 \%$ relataram não possuir quaisquer agravos.

\section{Discussão}

As organizações ao conhecerem a distribuição dos fatores de risco e de proteção para DCNT entre os colaboradores podem identificar situações de risco e direcionar ações de promoção da saúde e prevenção de agravos e doenças. Dentre as ações, merece destaque a avaliação nutricional na empresa a cada seis meses, atividade física na praia três vezes por semana por uma hora cada, com um educador físico; palestras educativas, distribuição anual de requisições para realização de exames laboratoriais e preventivos. Avaliações de saúde semestrais para acompanhamento da evolução de saúde e avaliação dos indicadores de monitoramento e resultados. O monitoramento dos fatores de risco para DCNT é importante para a abordagem populacional, indicando os benefícios das ações de promoção à saúde ${ }^{14}$.

Os resultados deste estudo demonstram que, em geral, a amostra avaliada apresenta perfil de risco intermediário para as DCNT. Porém, considera-se que tal variável é insatisfatória para determinar a ausência de risco cardiovascular entre os indivíduos que compuseram a amostra, uma vez que os mesmos apresentam perfil alimentar inadequado, bem como apresentaram um IMC acima do peso, o que aponta para um preditor de risco, embora, este resultado assemelhese com os achados da população adulta brasileira, a qual apresenta alta prevalência de sobrepeso e obesidade ${ }^{15}$.

Os níveis séricos de colesterol e triglicerídeos se elevam em função do consumo alimentar aumentado de colesterol, de carboidratos, de ácidos graxos saturados, de ácidos graxos trans e de excessiva quantidade de calorias. Por isso a seleção adequada destes itens poderá contribuir de maneira eficaz no controle das dislipidemias ${ }^{16}$. 
O sedentarismo, a adesão a uma dieta ocidentalizada, com hábitos alimentares ruins tem contribuído para 0 aumento da população obesa ${ }^{17}$. A obesidade representa o maior agravante de saúde e é vista como a doença crônicodegenerativa que mais afeta a população ${ }^{16,18}$.

Entretanto, destaca-se que o IMC é considerado por diversos autores como indicador importante para o risco cardiovascular, mas que apresenta algumas limitações, fato que dificulta a estimativa precisa de tal risco, haja vista que ao se utilizar este método tem-se uma dificuldade de diferenciação entre o tecido adiposo e o muscular dos indivíduos, com uma massa muscular aumentada, por exemplo, resultando em uma classificação inadequada para risco cardiovascular ${ }^{19}$.

Os achados evidenciaram alta prevalência de excesso de peso (61\%), tabagismo (2\%), etilismo (58\%), sedentarismo (72\%) e alimentação não saudável (83\%). Nas doenças já instaladas evidenciou-se HAS em 10\% dos entrevistados, 11\% com dislipidemia, $7 \%$ têm problemas respiratórios, 2,8\% são diabéticos e $0,8 \%$ tem depressão.

Os valores observados quanto aos indivíduos hipertensos foram semelhantes aos encontrados em outro estudo epidemiológico, o qual demonstrou taxa de prevalência em torno de $20 \%$, sem distinção por sexo, mas com evidente tendência de aumento com a idade ${ }^{20}$.

A HAS é apontada como um dos mais relevantes fatores de risco cardiovascular, uma vez que está relacionada à ocorrência de uma diversidade de DCV, e responde por cerca de $40 \%$ dos óbitos por acidente vascular encefálico (AVE), $25 \%$ dos ocorridos por doenças arteriais coronarianas e quando associada ao DM, estima-se que seja responsável por metade dos casos de desenvolvimento de insuficiência renal terminal ${ }^{2}$.

Dos $72 \%$ casos de óbito no Brasil, em 2005, tiveram como causas as DCNT. Desse total, $45 \%$ foram consideradas mortes prematuras, ocorridas antes dos 70 anos. Ainda segundo a Organização Mundial da Saúde (OMS), 80\% dos casos de DM2, problemas prematuros do coração e AVC, bem como $40 \%$ dos casos de câncer, podem ser evitados, se for adotada uma alimentação adequada, hábitos regulares de atividade física e redução do uso do tabaco²1.

Apesar de apresentar perfil de risco negativo para tabagismo e a população mínima possuírem já doenças crônicas, considerando-se uma elevação de mais da metade da população consumirem bebidas alcoólicas, a amostra avaliada apresenta perfil alimentar e inatividade física de alto risco, haja vista os hábitos alimentares inadequados observados, que certamente são influenciados pelo ritmo de vida adotado pela sociedade, apresentando-se como importante preditor de risco para doenças crônicas e estando comumente associada à elevação dos índices de sobrepeso e obesidade na população ${ }^{22}$.

Os dados encontrados neste estudo, em relação à alimentação inadequada, são compatíveis com dados da Pesquisa de Orçamentos Familiares (POF) 2008-2009, realizada pelo Instituto Brasileiro de Geografia e Estatística (IBGE) em parceria com o MS em 55.970 domicílios brasileiros. Essa pesquisa revelou que $90 \%$ dos avaliados apresentou consumo de frutas em quantidades menores do que é recomendado pelo MS, que é de cinco porções de frutas diariamente. A pesquisa revelou outro dado preocupante, haja vista que dos 260 indivíduos avaliados, $21,9 \%$ relataram não consumir frutas diariamente, fato que contribui para a vulnerabilidade dos indivíduos no que se refere ao acometimento por DCNT, como é o caso das DCV ${ }^{15}$.

Na população estudada, 26 colaboradores apresentaram HAS, representando $10 \%$ do total dos avaliados. A HAS, descrita como uma síndrome multifatorial e que costuma não apresentar sintomas, é comumente determinada pela presença de níveis de pressão arterial (PA) permanentemente elevados, em que se identifica valores de Pressão Arterial Sistólica (PAS) maiores que $140 \mathrm{mmHg}$ ou de Pressão Arterial Diastólica (PAD) maiores que $90 \mathrm{mmHg}$, sendo necessária a realização de no mínimo três aferições com um intervalo de tempo de uma semana entre as aferições para se estabelecer 0 diagnóstico de HAS. Esta doença encontra-se comumente associada a alterações metabólicas, funcionais e estruturais do encéfalo, coração, vasos sanguíneos e rins, resultando no desenvolvimento de DCV com desfechos que podem apresentarse de forma fatal ou não ${ }^{23}$.

Na relação dos avaliados, $3 \%$ são diabéticos. O DM compreende um relevante problema de saúde mundial que possui incidência crescente e alta morbimortalidade, resultando na perda significante da QV do indivíduo. Dados do MS indicam que o DM figura entre as 10 principais causas de morte na população brasileira (BRASIL, 2006b). Dados apontam que o DM foi responsável por 4,6 \% do total de mortes registradas no Brasil em $2007^{6}$.

$\mathrm{Na}$ Holanda, foi iniciado em 1998, o projeto Hartslag Limburg na região de Maastricht, que teve como objetivo reduzir a prevalência de DCNT, encorajando os habitantes a aumentarem a atividade física, a reduzirem a ingestão de gordura, e acessarem o tabagismo ${ }^{8}$. Desde 2002, a OMS está envolvida no esforço mundial de priorizar a vigilância das DCNT, com ênfase nos principais fatores de risco tais como o tabagismo, a HAS, a alimentação inadequada, a obesidade, o sedentarismo, entre outros. Diante das experiências exitosas em vários países na redução da morbimortalidade por DCNT por meio da vigilância e das ações integradas sobre os fatores de risco ${ }^{24}$. 
A síndrome metabólica, caracterizada pela associação de HAS, obesidade abdominal, intolerância à glicose, dislipidemias, apresenta índices elevados entre a população brasileira, incluindo a população jovem. Por outro lado, alterações no estilo de vida, como dieta adequada, prática regular de atividade física e abandono do tabagismo, podem aumentar a qualidade e a expectativa de vida dos indivíduos ${ }^{25-6}$.

A adesão à dieta, às correções no estilo de vida - perda de peso, atividade física e cessação do tabagismo. Essas mudanças de comportamentos adequados são fundamentais para a prevenção das $D C N T^{16}$.

$\mathrm{O}$ aumento da incidência desse tipo de doença no país gera impacto em muitos setores, como no econômico, uma vez que os cuidados com a doença crônica envolvem o uso de medicações e hospitalizações. Também se têm mudanças na organização social, alterando-se rotinas de trabalho, organização de tarefas, estrutura de locais públicos, aumento do número de aposentadorias por invalidez, entre outras coisas; além de efeitos na organização da família, com a reestruturação de papéis, criação de novas rotinas, possíveis mudanças econômicas; em aspectos mais individuais, como passar a conviver com mudanças e limites postos pela doença; e na própria cultura, que contemplará novos hábitos, significados compartilhados e a criação de novos espaços para aqueles que têm uma doença ${ }^{1,7}$.

A estruturação desses programas não deve esbarrar no estabelecimento de metas inalcançáveis. Cabe ressaltar que as metas preconizadas nos principais estudos para prevenção de doenças crônicas não são tão rigorosas que não possam ser atingidas. Ha evidências convincentes de que pequena redução de peso ( $5 \%$ do peso inicial), atividade física (150 minutos na semana) e hábitos alimentares saudáveis (> $15 \mathrm{~g} / 1.000 \mathrm{kcal}$ de fibras e $<10 \%$ de gorduras saturadas) promovem significativos benefícios cardiometabólicos. 0 alcance de apenas uma das metas já resulta em redução de risco; hipoteticamente, atingindo o conjunto das mudanças propostas, cerca de $90 \%$ dos casos seriam prevenidos ${ }^{27}$.

Ante aos achados, ressalta-se a "necessidade de reunir estas informações para a construção de políticas institucionais de promoção, prevenção, recuperação da saúde por meio de ações educativas"28:15.

\section{Considerações Finais}

Foi possivel constatar que os colaboradores estão em risco para o desenvolvimento de DCNT, pois muitos são os fatores relacionados.

Os principais fatores ambientais modificáveis das DCNT são os hábitos alimentares inadequados, o sedentarismo e a obesidade, associados às mudanças no estilo de vida como a cessação do tabagismo e o controle do stress. Dentro da prevenção, o monitoramento da prevalência dos fatores de risco para DCNT, especialmente os de natureza comportamental permitem, por meio das evidências observadas, a implementação de ações preventivas com maior custo-efetividade.

Portanto, programar ações de atenção à saúde e prevenção de doenças para colaboradores e beneficiários é o caminho eficaz para uma redução nos custos associados com o cuidado à saúde e absenteísmo, além de melhorias na saúde dos trabalhadores, ocasionando melhor QV. Mudanças sustentadas de comportamentos de risco estão entre os maiores desafios dos profissionais e dos órgãos e saúde.

Os resultados deste estudo permitem direcionar sobre novos caminhos a percorrer na promoção da saúde como estratégia de redução de doenças. Deste modo, não apenas visando redução de custos assistenciais das operadoras de planos de saúde, mas visando primordialmente a QV dos participantes desenvolvendo a consciência de sua responsabilidade e autonomia sobre o processo saúde-doença.

\section{Referências}

1. Duncan BB, Chor D, Aquino EML, Bensenor IM, Mill JG, Schmidt MI et al . Doenças crônicas não transmissíveis no Brasil: prioridade para enfrentamento e investigação. Rev Saúde Pública. 2012;46(1):126-34.

2. World Health Organization. Global status report on non communicable diseases 2010. Geneva: WHO, 2011.

3. Malta DC, Morais Neto, OL; Silva Júnior JB. Apresentação do plano de ações estratégicas para o enfrentamento das doenças crônicas não transmissíveis no Brasil, 2011 a 2022. Epidemiol Serv Saúde. 2011;20(4):425-38.

4. Schmidt MI, Duncan BB. O enfrentamento das doenças crônicas não transmissíveis: um desafio para a sociedade brasileira. Epidemiol. Serv Saúde. 2011;20(4):421-3. 
5. Orlando R, Pinheiro TLF, Volkweis DSH, Colussi EL. Avaliação da alimentação e sua relação com as doenças crônicas não transmissíveis de um grupo de idosos de um município da região norte do Estado do RS. Rev Enferm Frederico Westphalen. 2011;6(7):203-17.

6 Brasil. Ministério da Saúde. Secretaria de Vigilância em Saúde. Secretaria de Gestão Estratégica e Participativa. Vigitel Brasil 2010: vigilância de fatores de risco e proteção para doenças crônicas por inquérito telefônico. Brasília: MS; 2011. 7. Andrade JC. Fatores associados à hipertensão arterial e ao excesso de peso em trabalhadores de uma indústria metalúrgica [Dissertação]. Escola de Nutrição, Universidade Federal da Bahia, Salvador, BA, 2012.

8. Ribeiro AG, Cotta RMM, Ribeiro SMR. A Promoção da Saúde e a Prevenção Integrada dos Fatores de Risco para Doenças Cardiovasculares. Ciênc Saúde Coletiva. 2012;17(1):7-17.

9. Brasil. Ministério da Saúde. Organização Pan-Americana da Saúde. Doenças crônico-degenerativas e obesidade: estratégia mundial sobre alimentação saudável, atividade física e saúde. Brasília: OPAS; 2003.

10. Malta DC, Cezario AC, Moura L. A construção da vigilância e prevenção das doenças crônicas não transmissíveis no contexto do Sistema Único de Saúde. Epidemiol Serv Saúde. 2006;15(3):47-65.

11. Lima-Costa MF. Estilos de vida e uso de serviços preventivos de saúde entre adultos filiados ou não a plano privado de saúde (inquérito de saúde de Belo Horizonte). Ciênc Saúde Coletiva. 2004;9(4):857-64.

12. Unimed Norte Nordeste. Federação Interfederativa de Trabalhos Médicos. A cooperativa - 15 de setembro de 1977 [cited 2014 Aug 08]. Três Pontas (MG): UNIMED/FITM, 2014. Available from: http://unimednne.com.br/a-cooperativa/

13. Fontanella BJB, Luchesi BM, Saidel MGB, Ricas J, Turato ER, Melo DG. Amostragem em pesquisas qualitativas: proposta de procedimentos para constatar saturação teórica. Cad Saúde Pública. 2011;27(2):388-94.

14. Malta DC, Moura EC, Castro AM, Cruz DKA, Morais Neto OL, Monteiro CA. Padrão de atividade física em adultos brasileiros: resultados de um inquérito por entrevistas telefônicas, 2006. Epidemiol Serv Saúde. 2009;18(1):7-16.

15. Instituto Brasileiro de Geografia e Estatística. Pesquisa de Orçamento Familiar (POF) 2008- 2009: Antropometria e estado nutricional de crianças, adolescentes e adultos no Brasil. Rio de Janeiro: IBGE; 2010.

16. Xavier HT, Izar MC, Faria Neto JR, Assad MH, Rocha VZ, Sposito AC et al. V Diretriz Brasileira de Dislipidemias e Prevenção da Aterosclerose. Arq Bras Cardiol. 2013;101(4):1-20.

17. Guimarães AA, Bortolozo EAFQ, Lima DFR. Prevenção de fatores de risco para doenças cardiovasculares: programa de nutrição e prática de atividade física para servidores de uma universidade pública do estado do Paraná. Rev Eletr Fafit/Facic. 2013;4(1):10-8.

18. Wanderley EN, Ferreira VA. Obesidade: uma perspectiva plural. Ciênc Saúde Coletiva. 2010;15(1):185-94.

19. Rezende FAC, Rosado LEFPL, Franceschinni SCC, Rosado GP, Ribeiro RCL. Aplicabilidade do índice de massa corporal na avaliação da gordura corporal. Rev Bras Med Esporte. 2010;16(2):90-4.

20. Passos VMA, Assis TD, Barreto SM. Hipertensão arterial no Brasil: estimativa de prevalência a partir de estudos de base populacional. Serv Saúde. 2006;15(1);35-45.

21. World Health Organization. Global health risks: mortality and burden of disease attributable to selected major risks. Geneva: WHO; 2009.

22. Silva LS, Cotta RMM, Rosa COB. Estratégias de promoção da saúde e prevenção primária para enfrentamento das doenças crônicas: revisão sistemática. Rev Panam Salud Publica. 2013;34(5):343-50.

23. Sociedade Brasileira de Cardiologia. Sociedade Brasileira de Hipertensão. Sociedade Brasileira de Nefrologia. VI Diretrizes Brasileiras de Hipertensão. Arq Bras Cardiol. 2010;95(1 supl.1):1-51.

24. World Health Organization. Global estimate of the burden of disease from second-hand smoke. Geneva: WHO; 2010. 25. Ciolac EG, Guimarães GV. Exercício físico e síndrome metabólica. Rev Bras Med Esporte. 2004;10(4):48-52.

26. Pimenta AM, Gazzinelli A, Velásquez-Meléndez G. Prevalência da síndrome metabólica e seus fatores associados em área rural de Minas Gerais (MG, Brasil). Ciênc Saúde Coletiva. 2011;16(7): 3297-306.

27. Mozaffarian D, Kamineni A, Carnethon M, Djoussê L, MukamalKJ, Siscovick D. Lifestyle risk factors and new-onset diabetes mellitus in older adults: the Cardiovascular Health Study. Arch Intern Med. 2009;169(8):798-807.

28. Moura AL, Haddad MCL, Dalmas JC, Martins JT, Pissinati PSC. Avaliação do risco cardiovascular em trabalhadores de um campus universitário público. Saúde (Santa Maria). 2016;42(2):10-7. 


\section{Veronilde da Silva Oliveira}

Endereço para correspondência - Rua: Severino Soares, SN, Q13, L08, Condomínio Vilas do Lago, CEP: 58701-380, Patos, PB, Brasil.

E-mail: veraenfe@hotmail.com Lattes: http://lattes.cnpq.br/9784511556032462

Paula Christianne Gomes Gouveia Souto Maia - pcggsm@gmail.com Joselito Santos - jslito2012@gmail.com

Milena Nunes Alves de Sousa - minualsa@hotmail.com

Enviado em 02 de setembro de 2016. Aceito em 21 de novembro de 2016. 